\title{
Hypoglycemic Episodes Due to Synergistic Effect of Drugs in a Woman with Diabetes and Rheumatoid Arthritis
}

ISSN: 2578-0263

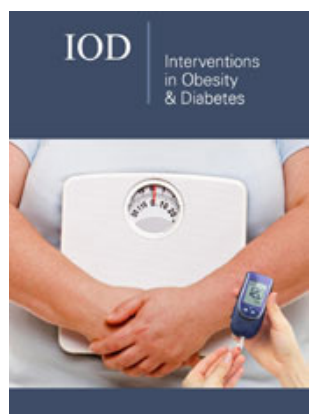

*Corresponding author: Argyrios V Tsakalis, Internal Medicine Outpatient Clinic, Health Center of Kastoria, Greek National Health System, Aristotle University of Thessaloniki, Greece

Submission: 望 April 24, 2020

Published: 海 May 15, 2020

Volume 4 - Issue 1

How tocitethisarticle:ArgyriosVTsakalis. Hypoglycemic Episodes Due to Synergistic Effect of Drugs in a Woman with Diabetes and Rheumatoid Arthritis. Interventions Obes Diabetes 4(1). IOD.000580. 2020 DOI: $10.31031 /$ IOD.2020.04.000580

Copyright@ Argyrios V Tsakalis, This article is distributed under the terms of the Creative Commons Attribution 4.0 International License, which permits unrestricted use and redistribution provided that the original author and source are credited.

\section{Argyrios V Tsakalis*}

Internal Medicine Outpatient Clinic, Health Center of Kastoria, Greek National Health System, Aristotle University of Thessaloniki, Greece

\begin{abstract}
Hydroxychloroquine (HCQ) and Leflunomide (LE) are agents that are commonly used to treat rheumatoid arthritis. Type 2 Diabetes (DM2) patients are in increased risk of hypoglycemia. The present report documents a case of repeated dawn hypoglycemic episodes due to concomitant use of HCQ LE and Repaglinide in a patient with Rheumatoid arthritis and DM2.
\end{abstract}

Keywords: Hydroxychloroquine, Leflunomide, Repaglinide, Hypoglycemia, Type 2 Diabetes, Rheumatoid arthritis

Abbreviations: HCQ: Hydroxy Chloro Quine; LEF: Leflunomide; DM2: Type 2 Diabetes; RA: Rheumatoid Arthritis; RE: Repaglinide; HYPO: Hypoglycemia; SITA: Sitagliptin

\section{Introduction}

Hydroxychloroquine (HCQ) is an antimalarial drug with anti-inflammatory properties that is employed in rheumatic diseases. Remarkable glucose lowering side effect of HCQ in non diabetic and diabetic patients is reported in literature [1-3]. Drug interactions can sometimes provoke to patients synergistic unexpected disturbing side effects; this is our case.

\section{Case Presentation}

A 49 year-old woman was first seen in our internal medicine outpatient clinic 3 months ago. Her past medical history included diabetes mellitus type 2 (DM2) for 5 years, seronegative rheumatoid arthritis (RA) for 4 years and hypertension. Her long-standing medications at that time included leflunomide (LE) 10mg daily, HCQ 200mg twice a day, prednisone $10 \mathrm{mg}$ daily, irbesartan $150 \mathrm{mg}$ daily, sitagliptin (SITA) 100mg daily, metformin $1000 \mathrm{mg}$ twice a day. Four months before our meeting her hemoglobin A1c was 5, 8\%, while it raised to $8 \%$ at her last control 10 days before she came to us. After the last result she referred to her general practitioner and he added SITA $100 \mathrm{mg}$ to her previous antidiabetic treatment that included only metformin $1000 \mathrm{mg}$ twice a day (morning and dinner time). After a week she referred to out clinic worried due to some recent high blood glucose (BG) levels during the morning and persistent high during the day. She referred no symptoms of hypoglycemia (HYPO) at that time. After controlling her glucometer data I confirmed the high BG levels during the day. Repaglinide (RE) 1mg twice a day before lunch and dinner was administered to her, while she continued also her previous medications. A schedule with three times per day BG measurement for a week was given to the patient prior to discharge. Our next appointment was programmed after a week.

The patient returned to our clinic reporting episodes of HYPO every morning. She reported glucometer readings approximating $50-65 \mathrm{mg} / \mathrm{dl}$ during these events. Instead, her glucometer reading were high normal during the day. Her HYPO symptoms included diaphoresis, hunger, anxiety and tremulousness. She reported no alcohol intake neither modification of diet habits during the last weeks. After my advice she discontinued her evening RE 1mg dose, while I transferred her second metformin dose uptake noontime instead of dinnertime. 
She continued her other long-standing medications. A schedule with three times per day BG measurement for a week was given to the patient prior to discharge. Our next appointment was programmed after a week. She returned on her appointment to our clinic reporting still, but milder HYPO episodes. Her glucometer readings were approximating $60-75 \mathrm{mg} / \mathrm{dl}$ during these events. Her glucometer reading were high normal during the day. SITA's dosage was decreased to $50 \mathrm{mg}$ daily after breakfast, while her noontime metformin uptake was decreased to $850 \mathrm{mg}$. She continued her other long-standing medications. A schedule with frequent BG measurement was given to the patient while our next appointment was programmed after 15 days. At our visit, she reported random fasting finger sticks in the $110-120 \mathrm{mg} / \mathrm{dl}$ range, and random sticks 2 hours after meal in the $140-180 \mathrm{mg} / \mathrm{dl}$ range. She reported no HYPO episodes. She was discharged with the same long-standing medications, while a schedule with daily BG measurement was given to her and the next appointment was arranged after 3 months.

\section{Discussion}

Diabetic patients are prone to HYPO episodes. In our case, to a patient that was taking sitagliptin and metformin (drugs that rarely cause HYPO when administered as the only therapy), RE was also added in order to control hyperglycemia. Her long-standing medications also included LEF and HCQ. Glucose lowering side effect of HCQ in diabetic patients is reported in literature $[1,3]$ Although the HCQ mechanism has not been clarified yet, increased insulin sensitivity and beta cell function [4-8] as well as decreased insulin degradation $[3,6,8]$, is suggested to contribute to the reduction in serum glucose levels. Furthermore, after the HYPO episodes I proceeded to an extensive control of Summary of Product Characteristics (SPC) of all medications the patient was taking. This patient was taking the anti-rheumatic drug LE, while RE was added in order to optimize glycemic control of her diabetes. As is reported to LE's SPC, its concomitant administration with RE, increases RE's Cmax (maximum concentration of the drug) and AUC (Area under the curve that indicates the drug's concentration in blood plasma) by 1,7 and 2,4 times respectively. This, according LE's SPC, occurs because LE is an "in vivo" Cytochrome P4502C8 inhibitor, meanwhile RE is degradated through this enzyme. To this patient the concomitant administration of HCQ LE and RE to a DM2 patient led to recurrent HYPO episodes during the night and morning, even if the patient had high BG levels days before RE administration. The HYPO episodes persisted even when RE and Metformin dinnertime dose were discontinued. The HYPO's vanished only when patient's previous antidiabetic treatment was drastically reduced and all dinnertime antidiabetic drugs were discontinued.

Diabetic patients are prone to HYPOS. Clinicians prescribing to them multiple drugs for other diseases should be aware that drug interactions can provoke to these patients synergistic unexpected disturbing side effects. Moreover, these patients should monitor closely their BG levels in order to prevent unexpected HYPO episodes.

\section{References}

1. Shojania K, Koehler BE, Elliott T (1999) Hypoglycemia induced by hydroxychloroquine in a type II diabetic treated for polyarthritis. J Rheumatol 26(1): 195-196.

2. Cansu DU, Korkmaz C (2008) Hypoglycaemia induced by hydroxychloroquine in a non-diabetic patient treated for RA. Rheumatology (Oxford) 47(3): 378-379.

3. Winter E, Schrander van der Meer A, Eustatia Rutten C, Janssen M (2011) Hydroxychloroquine as a glucose lowering drug. BMJ Case Rep: bcr0620114393.

4. Wasko M, McClure C, Kelsey S, Huber K, Orchard T, et al. (2015) Antidiabetogenic effects of hydroxychloroquine on insulin sensitivity and beta cell function: A randomised trial. Diabetologia 58(10): 23362343.

5. Bevan AP, Christensen JR, Tikerpae J, Smith GD (1995) Chloroquine augments the binding of insulin to its receptor. Biochem J 311(Pt 3): 787-795.

6. Opara E, Van Haeften $T$ (1987) Use of chloroquine in adipocyte in insulin binding. Diabetes 36: 160A.

7. Asamoah KA, Robb DA, Furman BL (1990) Chronic chloroquine treatment enhances insulin release in rats. Diabetes Res Clin Pract 9(3): 273-278.

8. Kang L, Mikuls T, O Dell JR (2009) Hydroxychloroquine: A diabetic drug in disguise? BMJ Case Rep.

For possible submissions Click below:

Submit Article 